\title{
Cold gas as an ice diagnostic toward low mass protostars ${ }^{\star}$
}

\author{
K. I. Öberg ${ }^{1}$, S. Bottinelli ${ }^{1}$, and E. F. van Dishoeck ${ }^{1,2}$ \\ 1 Leiden Observatory, Leiden University, PO Box 9513, 2300 RA Leiden, The Netherlands \\ e-mail: oberg@strw. leidenuniv.nl \\ 2 Max-Planck-Institut für extraterrestrische Physik (MPE), Giessenbachstraat 1, 85748 Garching, Germany \\ Received 27 October 2008 / Accepted 22 December 2008
}

\begin{abstract}
Context. Up to $90 \%$ of the chemical reactions during star formation occurs on ice surfaces, probably including the formation of complex organics. Only the most abundant ice species are however observed directly by infrared spectroscopy.

Aims. This study aims to develop an indirect observational method of ices based on non-thermal ice desorption in the colder part of protostellar envelopes.

Methods. The IRAM $30 \mathrm{~m}$ telescope was employed to observe two molecules that can be detected both in the gas and the ice, $\mathrm{CH}_{3} \mathrm{OH}$ and HNCO, toward 4 low mass embedded protostars. Their respective gas-phase column densities are determined using rotational diagrams. The relationship between ice and gas phase abundances is subsequently determined.

Results. The observed gas and ice abundances span several orders of magnitude. Most of the $\mathrm{CH}_{3} \mathrm{OH}$ and $\mathrm{HNCO}$ gas along the lines of sight is inferred to be quiescent from the measured line widths and the derived excitation temperatures, and hence not affected by thermal desorption close to the protostar or in outflow shocks. The measured gas to ice ratio of $\sim 10^{-4}$ agrees well with model predictions for non-thermal desorption under cold envelope conditions and there is a tentative correlation between ice and gas phase abundances. This indicates that non-thermal desorption products can serve as a signature of the ice composition. A larger sample is however necessary to provide a conclusive proof of concept.
\end{abstract}

Key words. astrochemistry - molecular processes - circumstellar matter - ISM: molecules - radio lines: ISM - molecular data

\section{Introduction}

In cold pre-stellar cores, more than $90 \%$ of all molecules, except for $\mathrm{H}_{2}$, are found in ices (Caselli et al. 1999; Bergin et al. 2002). These ices build up through accretion of atoms and molecules onto cold (sub)micron-sized silicate particles and subsequent hydrogenation to form e.g. $\mathrm{H}_{2} \mathrm{O}$ from $\mathrm{O}$ (Léger et al. 1985; Boogert \& Ehrenfreund 2004). Observations show that $\mathrm{H}_{2} \mathrm{O}$ is the main ice constituent in most lines of sight, with a typical abundance of $1 \times 10^{-4}$ with respect to $\mathrm{H}_{2}$, followed by $\mathrm{CO}, \mathrm{CO}_{2}$ and $\mathrm{CH}_{3} \mathrm{OH}$ (Gibb et al. 2004; Pontoppidan et al. 2004).

During star formation, these ices may be modified by interactions with cosmic rays, UV irradiation, and heating to form complex organic species (Garrod et al. 2008). Gas phase complex species have been observed toward several high and low mass protostars, so-called hot cores and corinos (Bisschop et al. 2007; Bottinelli et al. 2007). Whether these molecules are formed in the ice and subsequently evaporated, or formed in the hot gas phase from desorbed simpler ices such as $\mathrm{CH}_{3} \mathrm{OH}$ is still debated. This is not easily resolved because the abundances of the solid complex molecules are too low to be detected with infrared observations of ices even if they are present in the ice. Therefore, observing gas-phase abundances in the cold envelope may be the most robust constraint on complex ice processes available.

Experimental investigations have concluded that nonthermal desorption is efficient for several common ice molecules, such as $\mathrm{CO}, \mathrm{CO}_{2}$, and $\mathrm{H}_{2} \mathrm{O}$, with photodesorption yields of $\sim 10^{-3}$ per incident photon (Westley et al. 1995; Öberg et al. 2008, 2009). Photodesorption is possible inside cold

\footnotetext{
* Appendix A is only available in electronic form at http: //www . aanda.org
}

dark cloud cores and protostellar envelopes because of constant UV fields generated from cosmic ray interactions with $\mathrm{H}_{2}$ (Shen et al. 2004). Thus a small, but significant, part of the molecules formed in the ice should always be present in the gas phase. This explains observed abundances of gas phase $\mathrm{CH}_{3} \mathrm{OH}$ in translucent clouds, dark cloud cores and protostellar envelopes (Turner 1998; Maret et al. 2005; Requena-Torres et al. 2007). The amount of $\mathrm{CH}_{3} \mathrm{OH}$ gas observed in these environments suggests that complex molecules (e.g. methyl formate) that form in the ice should be observable in the gas phase due to ice photodesorption, if their abundance ratios with respect to $\mathrm{CH}_{3} \mathrm{OH}$ in the ice are the same as observed in hot cores and corinos.

For the first time, we combine infrared ice observations and millimeter gas observations for the same lines of sight to investigate the connection between ice and quiescent gas abundances. We focus on the only commonly observed ice components that have rotational transitions in the millimeter spectral range $-\mathrm{CH}_{3} \mathrm{OH}$ and $\mathrm{HNCO}$. The $\mathrm{CH}_{3} \mathrm{OH}$ ice abundances in low mass protostellar envelopes vary between $1-30 \%$ with respect to $\mathrm{H}_{2} \mathrm{O}$ ice (Boogert et al. 2008). It is also one of the most common hot corino gas phase molecules with typical abundances of $10^{-7}-10^{-6}$ with respect to $\mathrm{H}_{2}$. HNCO gas is also commonly detected in hot cores. Solid HNCO (in the form of $\mathrm{OCN}^{-}$) is only detected toward a few low mass protostars, but strict upper limits exist for more, resulting in an abundance span of an order of magnitude (van Broekhuizen et al. 2005). These large variations in ice abundances imply that $\mathrm{CH}_{3} \mathrm{OH}$ and $\mathrm{HNCO}$ are appealing test cases for our theory that quiescent complex gas abundances reflect the composition of the co-existing ice mantles, under the assumption that $\mathrm{OCN}^{-}$is protonated during desorption. 
We have observed gas phase $\mathrm{CH}_{3} \mathrm{OH}$ and $\mathrm{HNCO}$ with the IRAM $30 \mathrm{~m}$ toward four low mass protostars for which $\mathrm{CH}_{3} \mathrm{OH}$ and $\mathrm{HNCO}$ ice detections or upper limits already exist. Two of these sources also have $\mathrm{OCN}^{-}$ice upper limits. These sources are complemented with literature values to constrain further the relationship between ice and gas phase abundances.

\section{Source selection}

The four sources IRAS 03254+3050, B1-b, L1489 IRS and SVS 4-5 were chosen from the "cores to disks" ( $c 2 d)$ sample of low mass protostars with ice detections (Boogert et al. 2008) to span $\mathrm{CH}_{3} \mathrm{OH}$ abundances of $4-25 \%$ with respect to $\mathrm{H}_{2} \mathrm{O}$. The $c 2 d$ sample partly overlaps with an earlier ground based survey using the VLT, for which the $\mathrm{OCN}^{-}$abundances and upper limits were determined (van Broekhuizen et al. 2005) and two of the sources have $\mathrm{OCN}^{-}$ice upper limits (Table 1).

According to the classification scheme of Lada \& Wilking (1984), all sources are embedded class 0/I sources with spectral energy distribution (SED) slopes in the mid-infrared 2-24 $\mu \mathrm{m}$ between 0.68 and 1.26. Their envelopes are of similar mass, as traced by the $\mathrm{H}_{2} \mathrm{O}$ ice abundance, with the possible exception of B1-b, which has a factor of 3 higher column density. Except for SVS 4-5 the sources are isolated on the scale of the IRAM $30 \mathrm{~m}$ beam. Both B1-b and L1489 IRS have however moderate outflows associated with them that may contribute to the detected lines (Jørgensen et al. 2006; Girart et al. 2002).

SVS 4 region is one of the densest nearby star-forming regions and SVS $4-5$ is located $\sim 20^{\prime \prime}$ away from the class 0 lowmass protostar SMM 4, which has a large envelope and an associated outflow. In addition, the young stellar objects SVS 4-2-12 are all located within $30^{\prime \prime}$ of SVS 4-5 and the emission from SVS 4-5 is probably contaminated by emission from its surroundings when observed with a beam of width as large as $24^{\prime \prime}$ (as is the case here). Despite these complications in interpreting the data, SVS 4-5 is included in the sample because of its unusually high $\mathrm{CH}_{3} \mathrm{OH}$ ice abundance, which was determined from ice mapping of the SVS 4 region by Pontoppidan et al. (2004).

\section{Observations}

The observations were carried out in March 2008 with the 30-m telescope of the Institut de RadioAstronomie Millimétrique (IRAM). The positions used for pointing are listed in Table 1. The line frequencies are taken from the JPL molecular database. Although the observations were centered on the protostars themselves, the choice of low excitation lines and relatively large beams ensures that the cold outer envelope is almost completely sampled. Specifically, we targeted $\mathrm{CH}_{3} \mathrm{OH}$ transitions with $E_{\text {up }}$, the energy of the upper level of the transition, between 7 and $100 \mathrm{~K}$. The HNCO lines were observed after the preliminary reduction of the $\mathrm{CH}_{3} \mathrm{OH}$ data, and because of its low excitation temperature we chose to observe two of the lowest lying HNCO transitions with $E_{\text {up }}$ of 15 and $19 \mathrm{~K}$. The observations were carried out using four different receiver settings with the frequency ranges shown in Table 2. Each receiver was connected to a unit of the autocorrelator, with spectral resolutions of 80 or $320 \mathrm{kHz}$ and bandwidths between 80 and $480 \mathrm{MHz}$, equivalent to a velocity resolution of $0.3,0.4$ and $0.2 \mathrm{~km} \mathrm{~s}^{-1}$ in settings 1,2 , and $3 / 4$, respectively. Typical system temperatures were $100-200 \mathrm{~K}, 200-500 \mathrm{~K}$, and $700-1000 \mathrm{~K}$, at 3, 2, and $1 \mathrm{~mm}$, respectively.
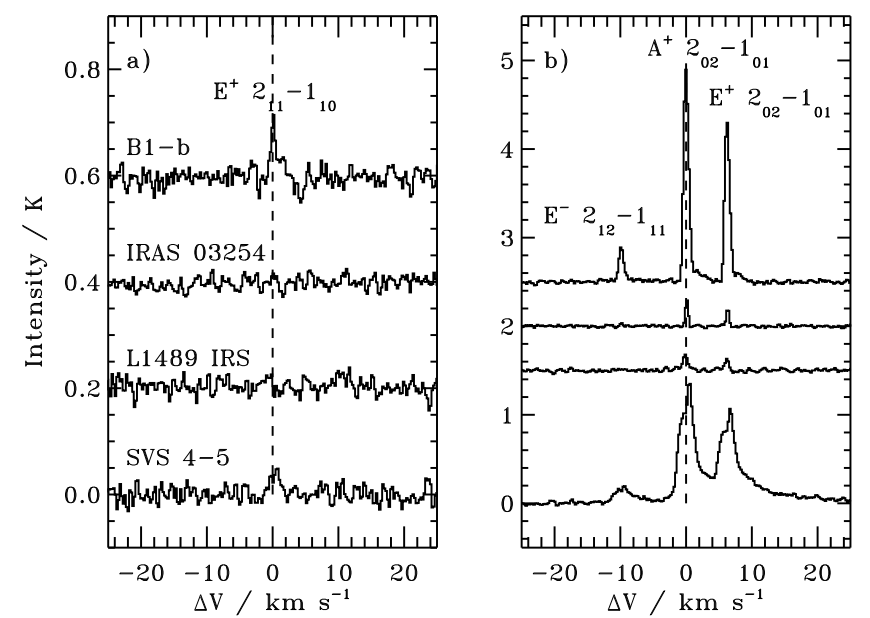

Fig. 1. The observed $\mathrm{CH}_{3} \mathrm{OH}$ lines in setting 1 toward the four low mass protostars plotted versus $\Delta V$, the deviation from the source $V_{\mathrm{lsr}}$. The data in the left panel are centered on a rest frequency of $96.756 \mathrm{GHz}$ and in the right panel on a rest frequency of $96.741 \mathrm{GHz}$.
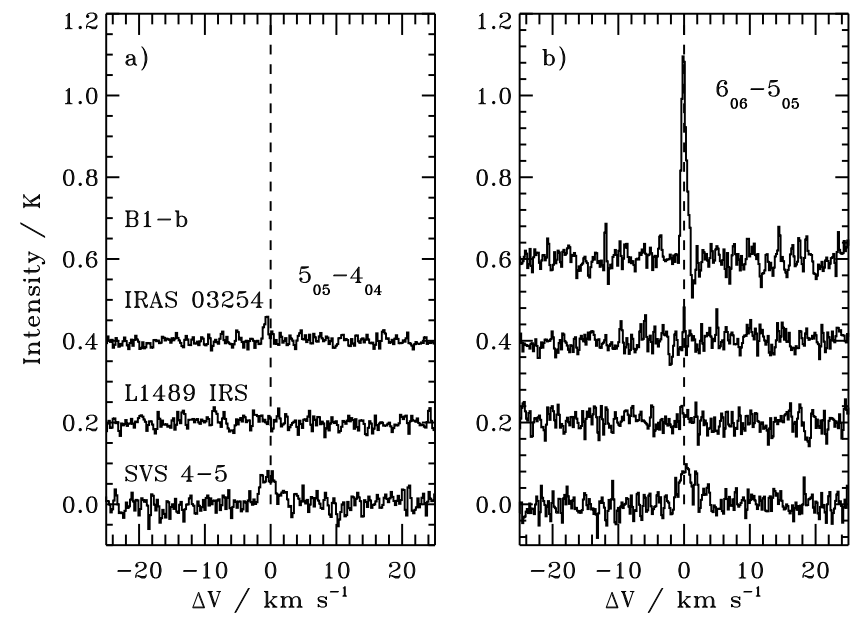

Fig. 2. The observed HNCO lines in setting 3 and 4 plotted versus $\Delta V$, the deviation from the source $V_{\mathrm{lsr}}$. The data in the left panel are centered on a rest frequency of $109.906 \mathrm{GHz}$ and in the right panel on $131.886 \mathrm{GHz}$.

All observations were carried out using wobbler switching with a $110^{\prime \prime}$ throw in azimuth. Pointing and focus were regularly checked using planets or strong quasars, providing a pointing accuracy of $3^{\prime \prime}$. All intensities reported in this paper are expressed in units of main-beam brightness temperature, which were converted from antenna temperatures using main beam efficiencies of 76,69 , and $50 \%$, at 3, 2, and $1 \mathrm{~mm}$. At these wavelengths, the beam sizes were 24,16 , and $10^{\prime \prime}$, respectively.

\section{Results}

Figure 1 shows the spectra derived for setting 1, which is the only setting in which $\mathrm{CH}_{3} \mathrm{OH}$ is detected. Figure 2 shows the obtained spectra in settings 3 and 4, with all targets observed in both settings except for B1-b. The observed lines were fitted with a single Gaussian to calculate the line widths and integrated intensities in Table 2. The Gaussian fits were restricted to exclude the wings observable for B1-b and SVS 4-5. The resulting line widths range from 0.4 to $4.0 \mathrm{~km} \mathrm{~s}^{-1}$, but three of the sources (B1-b, IRAS 03254, and L1489 IRS) consistently have line widths of below $1 \mathrm{~km} \mathrm{~s}^{-1}$ (Table 3). Coupled with the low 
Table 1. Targets with pointing positions and ice data.

\begin{tabular}{lcccccccc}
\hline \hline Source & RA & Dec & $\begin{array}{c}V_{\text {LSR }} \\
\mathrm{km} \mathrm{s}^{-1}\end{array}$ & $\begin{array}{c}\alpha \\
2-24 \mu \mathrm{m}\end{array}$ & Cloud & $\begin{array}{c}\mathrm{H}_{2} \mathrm{O} \text { col. dens. } \\
10^{18} \mathrm{~cm}^{-2}\end{array}$ & $\begin{array}{c}\mathrm{CH}_{3} \mathrm{OH} \\
\% \mathrm{H}_{2} \mathrm{O}\end{array}$ & $\begin{array}{c}\mathrm{HNCO} \\
\% \mathrm{H}_{2} \mathrm{O}\end{array}$ \\
\hline IRAS 03254+3050 & $03: 26: 37.45$ & $+30: 51: 27.9$ & 5.1 & 0.90 & Perseus & 3.66 & $<4.6$ & - \\
B1-b & $03: 33: 20.34$ & $+31: 07: 21.4$ & 6.5 & 0.68 & Perseus & 17.67 & 11.2 & - \\
L1489 IRS & $04: 04: 43.37$ & $+26: 18: 56.4$ & 7.1 & 1.10 & Taurus & 4.26 & 4.9 & $<0.06$ \\
SVS 4-5 & $18: 29: 57.59$ & $01: 13: 00.6$ & 7.8 & 1.26 & Serpens & 5.65 & 25.2 & $<0.27$ \\
\hline
\end{tabular}

Table 2. Observed frequencies and targeted molecules at the four different settings.

\begin{tabular}{|c|c|c|c|c|c|c|c|c|}
\hline \multirow{2}{*}{$\begin{array}{l}\text { Frequency } \\
\text { range }(\mathrm{GHz})\end{array}$} & \multirow{2}{*}{$\begin{array}{l}\text { Frequency } \\
(\mathrm{GHz})\end{array}$} & \multirow[t]{2}{*}{ Transition $^{a}$} & \multirow[t]{2}{*}{$E_{\mathrm{u}}(\mathrm{K})$} & \multirow[t]{2}{*}{ rms (mK) } & \multicolumn{4}{|c|}{ Integrated intensity [uncertainty] $\left(\mathrm{K} \mathrm{km} \mathrm{s}^{-1}\right)$} \\
\hline & & & & & IRAS $03254^{\circ}$ & B1-b & L1489 IRS & SVS 4-5 \\
\hline \multirow{5}{*}{$\begin{array}{l}\mathrm{CH}_{3} \mathrm{OH} \\
\text { 1) } 96.739-96.756\end{array}$} & & & & & & & & \\
\hline & $96.739\left(\mathrm{E}^{-}\right)$ & $212-1_{11}$ & 20.0 & $11-15$ & $0.11[0.03]$ & $1.65[0.22]$ & $0.10[0.03]$ & $1.47[0.33]$ \\
\hline & $96.741\left(\mathrm{~A}^{+}\right)$ & $2_{02}-1_{01}$ & 14.4 & & $0.16[0.04]$ & $2.18[0.21]$ & $0.17[0.05]$ & $2.72[0.58]$ \\
\hline & $96.745\left(\mathrm{E}^{+}\right)$ & $2_{02}-1_{01}$ & 27.5 & & 0.017 [0.017] & $0.37[0.20]$ & $0.024[0.024]$ & $0.64[0.39]$ \\
\hline & $96.756\left(\mathrm{E}^{+}\right)$ & $2_{11}-1_{10}$ & 35.4 & & $<0.01$ & $0.09[0.26]$ & $<0.024$ & $0.074[0.030]$ \\
\hline 2) $251.360-251.811$ & $251.738\left(\mathrm{~A}^{ \pm}\right)$ & $6_{33}-6_{24}$ & 98.6 & $28-40$ & $<0.033$ & - & $<0.079$ & $<0.26$ \\
\hline \multicolumn{9}{|l|}{$\mathrm{HNCO}$} \\
\hline 3) $109.872-109.938$ & 109.906 & $5_{05}$ & 15.4 & & $0.038[0.030]$ & _ & $<0$. & $0.22[0.07]$ \\
\hline 4) $131.885-131.886$ & 131.886 & $6_{06}-5_{05}$ & 18.6 & $19-29$ & $<0.021$ & $0.43[0.06]$ & $<0.037$ & $0.22[0.07]$ \\
\hline
\end{tabular}

${ }^{a}$ The quantum numbers for the pure rotational transitions of $\mathrm{CH}_{3} \mathrm{OH}$ and $\mathrm{HNCO}$ are $J_{\mathrm{K}_{a} \mathrm{~K}_{\mathrm{c}}}$ and $J_{\mathrm{K}_{-1} \mathrm{~K}_{+1}}$, respectively.

excitation temperatures (below), we most likely probe the quiescent envelope rather than outflows or hot corinos. The fourth source SVS 4-5 has several emission components, reflecting the complexity of the SVS 4 region and containing contributions from non-quiescent gas in for example the nearby outflow from SMM 4. The typical envelope angular size for the source distance is $\sim 1^{\prime}$, which is larger than the largest beam size. Hence, we assume in the analysis that there is no beam dilution.

In Figs. 3 and 4, we use the rotational diagram method (Goldsmith \& Langer 1999) to derive rotational temperatures and column densities. The relations evident in the $\mathrm{CH}_{3} \mathrm{OH}$ diagrams are approximately linear, with a possible slight deviation for the one $\mathrm{CH}_{3} \mathrm{OH}$ A detection. A line is fitted to all $\mathrm{CH}_{3} \mathrm{OH}$ detections, with the assumption that the populations of $\mathrm{E}$ and $\mathrm{A}$ species are approximately equal. The $2 \sigma$ upper limits (derived from the rms in Table 2) are overplotted to enable us to ensure that they do not provide further constraints on the fitted line. Except for SVS 4-5, HNCO column densities were derived using the $\mathrm{CH}_{3} \mathrm{OH}$ rotational temperatures. The lower limit to the $\mathrm{CH}_{3} \mathrm{OH}$ temperature of $4 \mathrm{~K}$ was used for IRAS 03254 to accommodate the strict upper limit from setting 3 (Table 3 ). The resulting $\mathrm{CH}_{3} \mathrm{OH}$ and $\mathrm{HNCO}$ temperatures vary between 4 and $9 \mathrm{~K}$ and the $\mathrm{CH}_{3} \mathrm{OH}$ and $\mathrm{HNCO}$ column densities vary between $1.8-27 \times 10^{13}$ and $0.095-2.4 \times 10^{13} \mathrm{~cm}^{-2}$, respectively. $\mathrm{CH}_{3} \mathrm{OH}$ is easily sub-thermally excited at the expected densities in outer protostellar envelopes of approximately $10^{4} \mathrm{~cm}^{-3}$ and hence the rotational temperature cannot be directly translated into a kinetic temperature (Bachiller et al. 1995).

In previous studies, gas phase $\mathrm{CH}_{3} \mathrm{OH}$ was observed toward three other low mass protostars that were also observed by Spitzer to study ices (Boogert et al. 2008): Elias 29, R CrA 7A, and $\mathrm{B}$ (Table 3). The line widths imply that the observed $\mathrm{CH}_{3} \mathrm{OH}$ lines toward Elias 29 trace quiescent material, while those observed toward R CrA 7A and B do not.

Figure 5 shows the correlation between gas and ice abundances of $\mathrm{CH}_{3} \mathrm{OH}$ and $\mathrm{HNCO}$, including the literature sources. The abundances are with respect to the $\mathrm{H}_{2} \mathrm{O}$ ice column density, which was found to correlate well with the cold dust column density (Whittet et al. 2001). Hence this is a reasonable normalization factor for the lines of sight with quiescent gas,

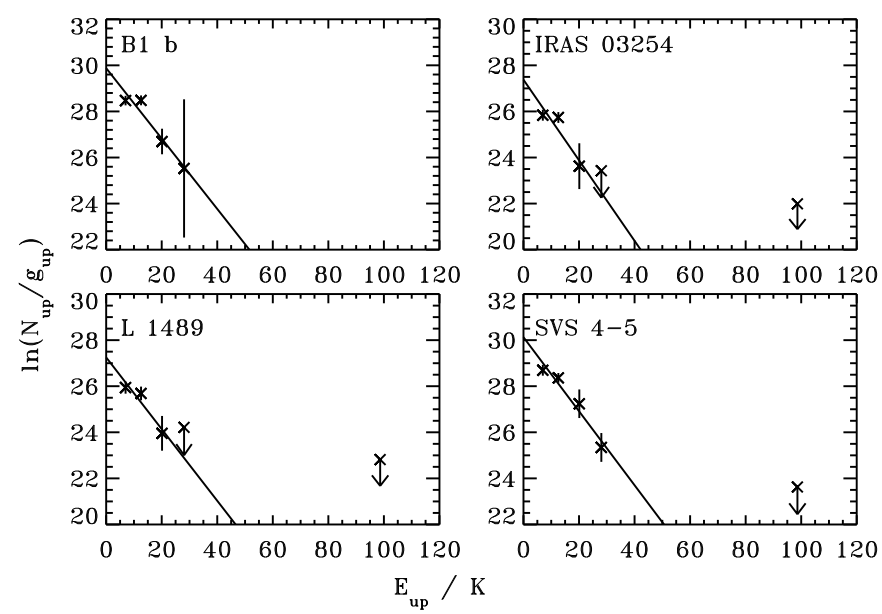

Fig. 3. $\mathrm{CH}_{3} \mathrm{OH}$ rotation diagrams including detections and upper limits.

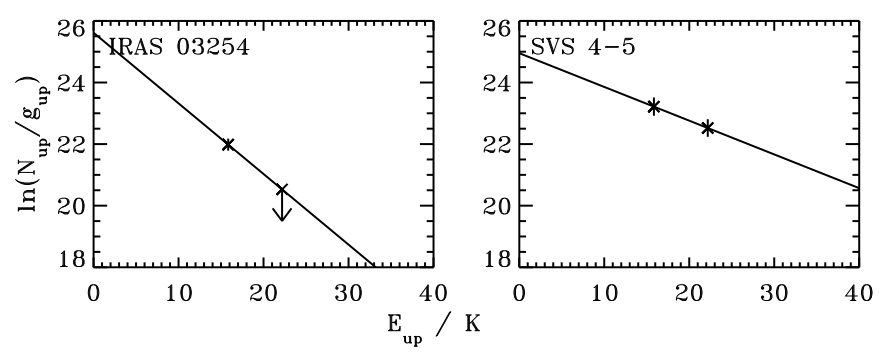

Fig. 4. HNCO rotation diagrams where both lines are detected or the upper limit is strict.

here defined to be line widths $\lesssim 1 \mathrm{~km} \mathrm{~s}^{-1}$, that originates in the cold envelope. It is not a priori a good normalizer for sources with non-quiescent emission, but for consistency the same normalization method is used for all sources. Figure 5 illustrates a possible correlation between the gas and ice abundances and upper limits for the quiescent sources - the correlation is not statistically significant due to the many upper limits. The two open triangles in the figure are the RCrA sources, whose higher ratio 
Table 3. The calculated temperatures and column densities.

\begin{tabular}{lcccc}
\hline \hline Source & Molecule & $\begin{array}{c}\text { Line width } \\
\left(\mathrm{km} \mathrm{s}^{-1}\right)\end{array}$ & $\begin{array}{c}T_{\text {rot }} \\
(\mathrm{K})\end{array}$ & $\begin{array}{c}N_{\mathrm{X}} \\
\left(\times 10^{13} \mathrm{~cm}^{-2}\right)\end{array}$ \\
\hline IRAS 03254 & $\mathrm{CH}_{3} \mathrm{OH}$ & $0.42-0.49$ & $6 \pm 2$ & $1.8 \pm 1.8$ \\
& $\mathrm{HNCO}$ & 0.77 & $\sim 4$ & $\sim 0.48$ \\
B1-b & $\mathrm{CH}_{3} \mathrm{OH}$ & $0.77-0.88$ & $7 \pm 1$ & $25 \pm 13$ \\
& $\mathrm{HNCO}^{2}$ & 0.86 & $\sim 7$ & $\sim 2.4$ \\
L1489 IRS & $\mathrm{CH}_{3} \mathrm{OH}$ & $0.75-0.97$ & $6 \pm 2$ & $1.8 \pm 1.3$ \\
& $\mathrm{HNCO}$ & - & $\sim 6$ & $<0.095$ \\
SVS 4-5 & $\mathrm{CH}_{3} \mathrm{OH}$ & $2.4-4.0$ & $7 \pm 1$ & $27 \pm 10$ \\
& $\mathrm{HNCO}^{2}$ & $2.6-2.9$ & $9 \pm 6$ & $0.38 \pm 0.38$ \\
\hline Elias 29 & $\mathrm{CH}_{3} \mathrm{OH}$ & 1.3 & $\sim 9$ & $\sim 0.73$ \\
R CrA 7A & $\mathrm{CH}_{3} \mathrm{OH}$ & $2.4-3.0$ & $18 \pm 2$ & $59 \pm 28$ \\
$\mathrm{R} \mathrm{CrA} \mathrm{7B}^{b}$ & $\mathrm{CH}_{3} \mathrm{OH}$ & $2.1-2.6$ & $19 \pm 1$ & $94 \pm 28$ \\
\hline
\end{tabular}

${ }^{a}$ Buckle \& Fuller (2002); ${ }^{b}$ derived from Schöier et al. (2006) using rotational diagrams.

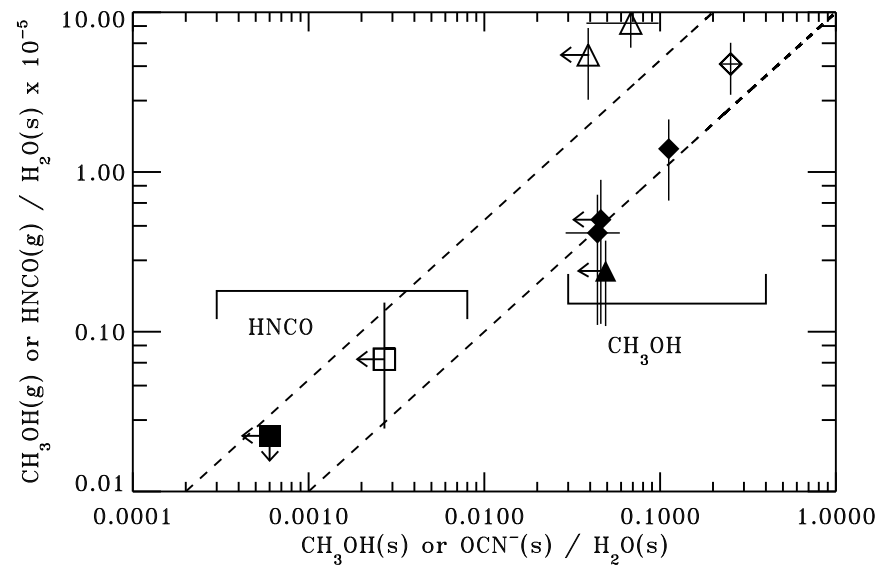

Fig. 5. The correlation between ice and gas phase abundances of $\mathrm{CH}_{3} \mathrm{OH}$ (diamonds and triangles) and HNCO (squares). The filled symbols represent quiescent gas $\left(<1.3 \mathrm{~km} \mathrm{~s}^{-1}\right)$ and the open symbols nonquiescent gas $\left(>2.4 \mathrm{~km} \mathrm{~s}^{-1}\right)$. In the case of $\mathrm{CH}_{3} \mathrm{OH}$, the diamonds represent measurements from this study and triangles from the literature. The dashed lines show constant $\mathrm{CH}_{3} \mathrm{OH}$ (gas) $/ \mathrm{CH}_{3} \mathrm{OH}$ (solid) or $\mathrm{HNCO}($ gas $) / \mathrm{OCN}^{-}$(solid) ratios of $1 \times 10^{-4}$ and $5 \times 10^{-4}$.

of gas to ice phase abundance can be attributed to an enhanced radiation field in the region (van Kempen 2008). The measured average gas to solid abundance ratio is $1.2 \times 10^{-4}$ for the quiescent gas. This probably underestimates the true gas to ice ratio because the absorption and emission observations differ, i.e., the gas phase observations probe on average less dense regions than the ice observations.

\section{Discussion}

Ice photodesorption predicts gas to ice ratios of $10^{-4}-10^{-3}$ (see Appendix) for typical photodesorption yields and envelope conditions. The measured gas to ice ratio in this study of $1.2 \times$ $10^{-4}$ agrees well with this prediction, when accounting for the fact that the measured ratio probably underestimates the true gas to ice ratio. The dashed lines in Fig. 5 further show that all quiescent detections and upper limits are consistent with gas to ice ratios of $(1-5) \times 10^{-4}$. This agreement and the narrowness of the line widths, supports the interpretation that the emission in these lines of sight originates in the cold, quiescent envelope. It also demonstrates that photodesorption alone is sufficient to release the observed ice into the gas, even though other nonthermal processes are not excluded since lack of experimental studies on most non-thermal desorption pathways prevents us from quantifying their relative importance. Gas phase reactions can however be excluded since there is no efficient gas phase reaction pathway to form either $\mathrm{CH}_{3} \mathrm{OH}$ or $\mathrm{HNCO}$ at the observed abundances (Garrod et al. 2007, Hassel private comm.).

The tentative correlation between gas and ice phase abundances in this pilot study supports the idea that it is possible to determine ice composition by observing the small fraction of the ice that is non-thermally released into the gas phase. To show conclusively that this method works, the size of the sample studied here must be increased and the uncertainty in the derived column densities must be reduced by observing more emission lines. It is also important to remember that until photodesorption data are available for all potential ice species there will be at least a factor of two uncertainty in ice composition estimates using this method due to the different break-up probabilities of different molecules during photodesorption (Öberg et al. 2008, 2009). Nonetheless, the method presented here represents a significant improvement on the current lack of observational tools to study complex ices in quiescent regions.

Acknowledgements. We thank Ruud Visser for stimulating discussions. Funding is provided by NOVA, the Netherlands Research School for Astronomy, the European Early Stage Training Network ("EARA" MEST-CT-2004-504604), a Netherlands Organisation for Scientific Research (NWO) Spinoza grant and the European Community's sixth Framework Programme under RadioNet (R113CT 2003 5058187).

\section{References}

Bachiller, R., Liechti, S., Walmsley, C. M., \& Colomer, F. 1995, A\&A, 295, L51 Bergin, E. A., Alves, J., Huard, T., \& Lada, C. J. 2002, ApJ, 570, L101 Bisschop, S. E., Jørgensen, J. K., van Dishoeck, E. F., \& de Wachter, E. B. M. 2007, A\&A, 465, 913

Boogert, A. C. A., \& Ehrenfreund, P. 2004, in Astrophysics of Dust, ed. A. N. Witt, G. C. Clayton, \& B. T. Draine, ASP Conf. Ser., 309, 547

Boogert, A. C. A., Pontoppidan, K. M., Knez, C., et al. 2008, ApJ, 678, 985 Bottinelli, S., Ceccarelli, C., Williams, J. P., \& Lefloch, B. 2007, A\&A, 463, 601 Buckle, J. V., \& Fuller, G. A. 2002, A\&A, 381, 77

Caselli, P., Walmsley, C. M., Tafalla, M., Dore, L., \& Myers, P. C. 1999, ApJ, 523, L165

Garrod, R. T., Wakelam, V., \& Herbst, E. 2007, A\&A, 467, 1103

Garrod, R. T., Widicus Weaver, S. L., \& Herbst, E. 2008, ArXiv e-prints, 803 Gibb, E. L., Whittet, D. C. B., Boogert, A. C. A., \& Tielens, A. G. G. M. 2004 , ApJS, 151, 35

Girart, J. M., Curiel, S., Rodríguez, L. F., \& Cantó, J. 2002, Rev. Mex. Astron. Astrofis., 38, 169

Goldsmith, P. F., \& Langer, W. D. 1999, ApJ, 517, 209

Jørgensen, J. K., Harvey, P. M., Evans, II, N. J., et al. 2006, ApJ, 645, 1246

Léger, A., Jura, M., \& Omont, A. 1985, A\&A, 144, 147

Maret, S., Ceccarelli, C., Tielens, A. G. G. M., et al. 2005, A\&A, 442, 527

Öberg, K. I., van Dishoeck, E. F., \& Linnartz, H. 2008 [arXiv:0809. 1333]

Öberg, K. I., Visser, R., van Dishoeck, E. F., \& Linnartz, H. 2009, ApJ, in press

Pontoppidan, K. M., van Dishoeck, E. F., \& Dartois, E. 2004, A\&A, 426, 925

Requena-Torres, M. A., Marcelino, N., Jiménez-Serra, I., et al. 2007, ApJ, 655, L37

Schöier, F. L., Jørgensen, J. K., Pontoppidan, K. M., \& Lundgren, A. A. 2006, A\&A, 454, L67

Shen, C. J., Greenberg, J. M., Schutte, W. A., \& van Dishoeck, E. F. 2004, A\&A, 415, 203

Turner, B. E. 1998, ApJ, 501, 731

van Broekhuizen, F. A., Pontoppidan, K. M., Fraser, H. J., \& van Dishoeck, E. F. 2005, A\&A, 441, 249

van Kempen, T. 2008, Ph.D. Thesis, Leiden University

Westley, M. S., Baragiola, R. A., Johnson, R. E., \& Baratta, G. A. 1995, Nature, 373,405

Whittet, D. C. B., Gerakines, P. A., Hough, J. H., \& Shenoy, S. S. 2001, ApJ, 547,872 


\section{Appendix A: Derivation of gas to ice ratios}

The gas to ice ratio for a particular species in a protostellar envelope can be estimated by assuming a steady-state between photodesorption and freeze-out:

$Y_{\mathrm{pd}} \times I_{\mathrm{UV}} \times \sigma_{\mathrm{gr}} \times f_{\mathrm{x}}=4.57 \times 10^{4} \times\left(\frac{T}{m_{\mathrm{x}}}\right)^{\frac{1}{2}} \times \sigma_{\mathrm{gr}} \times n_{\mathrm{x}}^{g}$

$f_{\mathrm{x}}=\frac{n_{\mathrm{x}}^{\mathrm{i}}}{n^{\mathrm{i}}}$

where $Y_{\mathrm{pd}}$ is the photodesorption yield set to be $(1-3) \times$ $10^{-3}$ photon $^{-1}$ from our experiments, $I_{\mathrm{UV}}$ is the cosmic-rayinduced UV field of $10^{4}$ photons $\mathrm{cm}^{-2} \mathrm{~s}^{-1}$ and $\sigma_{\mathrm{gr}}$ is the grain cross section. The cosmic-ray-induced UV flux assumes a cosmic ray ionization rate of $1.3 \times 10^{-17} \mathrm{~s}^{-1}$. Because photodesorption is a surface process, the photodesorption rate of species $\mathrm{X}$ depends on the fractional ice abundance $f_{\mathrm{x}}$, which is defined to be the ratio of the number density of species $\mathrm{x}$ in the ice, $n_{\mathrm{x}}^{\mathrm{i}}$, to the total ice number density, $n^{\mathrm{i}}$. The freeze-out rate of species $\mathrm{x}$ depends on the gas temperature $T$, which is set to $15 \mathrm{~K}$, the molecular weight $m_{\mathrm{x}}$, and the gas number density $n_{\mathrm{x}}^{\mathrm{g}}$. For an average molecular weight of 32 , this results in a gas phase abundance $n_{\mathrm{x}}^{\mathrm{g}} / n_{\mathrm{H}}$ of $(3-9) \times 10^{-4} f_{\mathrm{x}} / n_{\mathrm{H}}$. From this and an average total ice abundance $n^{\mathrm{i}} / n_{\mathrm{H}}$ of $10^{-4}$, the predicted gas to ice phase abundance ratio is:

$\frac{n_{\mathrm{x}}^{\mathrm{g}}}{n_{\mathrm{x}}^{\mathrm{i}}} \sim \frac{(3-9) \times 10^{-4} / n_{\mathrm{H}} \times f_{\mathrm{x}}}{n^{\mathrm{i}} / n_{\mathrm{H}} \times f_{\mathrm{x}}} \sim(3-9) / n_{\mathrm{H}}$
For a typical envelope density of $10^{4} \mathrm{~cm}^{-3}$, ice photodesorption hence predicts a gas to ice ratio of $10^{-4}-10^{-3}$. The derivation of a gas to ice ratio from observed cold gas emission lines and ice absorption features in the same line of sight is complicated by the fact that different regions can contribute by varying amounts. The emission features trace gas in the envelope and cloud both in front and behind the protostar, while the ice absorption features only trace envelope material directly in front of the protostar. The column is hence twice as long for the gas observations. This is probably more than compensated for by using beam averaged gas column densities, as is done in this study, because the large beam traces on average less dense material compared to the pencil beam of the ice absorption observations. Note also that the $\mathrm{CH}_{3} \mathrm{OH}$ ice abundance may vary between lower and higher density regions. To quantify a conversion factor between the observed and true gas to ice ratio requires detailed modeling of each source which is outside the scope of this study. Instead we here assume that the observed ratio is a lower limit to the true ratio. 